\title{
Electroacupuncture at the Baihui acupoint alleviates cognitive impairment and exerts neuroprotective effects by modulating the expression and processing of brain-derived neurotrophic factor in APP/PS1 transgenic mice
}

\author{
RUHUI LIN ${ }^{1,2^{*}}$, JIXIANG CHEN ${ }^{1,3^{*}},{\text { XIAOJIE } \text { LI }^{1}, \text { JINGJIE MAO }^{2}, \text { YUNAN WU }^{1} \text {, PEIYUAN ZHUO }}^{1}$, \\ YINZHENG ZHANG ${ }^{1}$, WEILIN LIU ${ }^{1,4}$, JIA HUANG $^{1,4}$, JING TAO $^{1,5}$ and LI-DIAN CHEN ${ }^{1,6}$ \\ ${ }^{1}$ College of Rehabilitation Medicine, Fujian University of Traditional Medicine, Fuzhou, Fujian 350122; \\ ${ }^{2}$ Academy of Integrative Medicine Biomedical Research Center, Fuzhou, Fujian 350122; ${ }^{3}$ Department of Rehabilitation Medicine, \\ Guangzhou First People's Hospital, Guangzhou, Guangdong 510180; ${ }^{4}$ Fujian Key Laboratory of Exercise Rehabilitation; \\ ${ }^{5}$ TCM Rehabilitation Research Center of SATCM, Fuzhou, Fujian 350122; ${ }^{6}$ Fujian Rehabilitation Tech Co-innovation Center, \\ Fujian University of Traditional Medicine, Fuzhou, Fujian 350122, P.R. China
}

Received April 1,2015; Accepted December 2, 2015

DOI: $10.3892 / \mathrm{mmr} .2015 .4751$

\begin{abstract}
Alzheimer's disease (AD) is a common human neurodegenerative disorder characterized by progressive deterioration of cognition and memory. Acupuncture at the Baihui (DU20) acupoint has long been used in China to clinically treat cognitive impairment. However, the precise mechanism underlying its neuroprotective effects remains to be elucidated. In the present study, electroacupuncture (EA) at the Baihui (DU20) acupoint was observed to markedly ameliorate cognitive impairments, reduce the aberrant overexpression of $\beta$-amyloid(1-42), and inhibit neuronal apoptosis in APP/PS1 mice. As brain-derived neurotrophic factor (BDNF) has been implicated in the pathogenesis of AD, the expression and processing of BDNF in APP/PS1 mice was investigated. EA at the Baihui (DU20) acupoint was indicated to significantly enhance the expression levels of mature BDNF and proBDNF in APP/PS1 mice. Furthermore, an increase in the $\mathrm{BDNF} /$ proBDNF ratio, upregulation of the expression levels of phosphorylated tropomyosin receptor kinase B and a decrease in the expression level of p75 neurotrophin receptor were also observed in the APP/PS1 mice. The present study demonstrates
\end{abstract}

Correspondence to: Dr Li-Dian Chen, College of Rehabilitation Medicine, Fujian University of Traditional Chinese Medicine, 1 Huatuo Road, Fuzhou, Fujian 350122, P.R. China

E-mail: cld@fjtcm.edu.cn

*Contributed equally

Key words: electroacupuncture, Baihui (DU20) acupoint, cognitive impairment, APP/PS1 transgenic mice, brain-derived neurotrophic factor the efficacy of EA at the Baihui (DU20) acupoint in the treatment of cognitive impairments in APP/PS1 transgenic mice. The present study hypothesized that modulation of BDNF expression and processing may be the underlying mechanism by which stimulation of the Baihui (DU20) acupoint exerts its neuroprotective effect.

\section{Introduction}

Alzheimer's disease (AD) is a prevalent neurodegenerative disease characterized by the progressive deterioration of cognition and memory $(1,2)$. Pathological features of AD include the accumulation of extracellular senile plaques predominantly composed of $\beta$-amyloid (A $\beta$ ) peptide, intracellular neurofibrillary tangles and neuronal loss, particularly in the hippocampus (3). Numerous previous studies have indicated that brain-derived neurotrophic factor (BDNF) is associated with the pathogenesis of AD. In 1991, Phillips et al (4) demonstrated a selective reduction in BDNF messenger (m)RNA expression levels in the hippocampi of patients with AD. Subsequent studies indicated that the precursor of BDNF (proBDNF), mature BDNF (mBDNF) and BDNF mRNA expression levels were decreased in individuals with $\mathrm{AD}$, and the levels of BDNF were positively correlated with cognitive measures, including the Global Cognitive Score and the Mini Mental State Examination score $(5,6)$. A community-based, prospective cohort study, which involved 2,131 dementia-free participants ( $56 \%$ women) aged $\geq 60$ years (age, $72 \pm 7$ years; mean \pm standard deviation), observed that increased serum BDNF levels were associated with a reduced risk for dementia and AD (7). These data suggest that BDNF is important in the pathogenesis of AD.

BDNF is synthesized as a precursor protein, proBDNF, which is post-translationally cleaved to produce mBDNF. This processing of the BDNF protein appears to be key in regulating its cellular function $(8,9)$. ProBDNF preferentially binds to the 
p75 neurotrophin receptor ( $\mathrm{p} 75^{\mathrm{NTR}}$ ) leading to downstream signaling, via signaling pathways involved in apoptosis, and facilitating long-term depression in the hippocampus (10). By contrast, cleaved mBDNF binds to the tropomyosin receptor kinase B (TrkB) receptor, promotes cell survival and facilitates certain forms of long-term potentiation (11). Previous studies have demonstrated that BDNF signaling exerts neuroprotective effects against $\mathrm{A} \beta$ peptide toxicity in vivo and in vitro $(12,13)$. However, it has also been reported that proBDNF suppresses the proliferation of hippocampal neurons in the dentate gyrus in AD rats and anti-proBDNF reverses the effects (14). Therefore, the processing of the BDNF protein may be important in AD.

Acupuncture at the Baihui (DU20) acupoint has long been used in China to treat cognitive impairment. It is located on a branch of the Du Meridian that runs over the head and is associated with cognitive function in traditional Chinese medicine. However, the efficacy of acupuncture at the Baihui (DU20) acupoint in animal models of AD and its underlying mechanism of action are not well understood. A previous study has demonstrated that electroacupuncture (EA) at the Baihui (DU20) and Shenting (DU24) acupoints ameliorates cognitive impairment in cerebral ischemia-reperfusion injured rats (15). Therefore, in the present study, the therapeutic efficacy of EA against cognitive deficits was evaluated in APP/PS1 transgenic mice, and the effects of BDNF expression and processing underlying the neuroprotective effects were investigated.

\section{Materials and methods}

Materials and reagents. Terminal deoxynucleotidyl transferase dUTP nick end labeling (TUNEL) assay kits (DeadEnd Fluorometric TUNEL System) were obtained from Promega Corporation (Madison, WI, USA) and 3,3'-diaminobenzidine (DAB) kits (Kit-0017) were purchased from Fuzhou Maixin Biotech, Co., Ltd. (Fuzhou, China). Polyclonal rabbit anti-A $\beta(1-42)$ (1:200; cat. no. ab10148) and monoclonal rabbit anti-BDNF (1:1,000; cat. no. ab108319) were obtained from Abcam (Cambridge, UK). Polyclonal rabbit anti-proBDNF (1:400; cat. no. ANT-006) was obtained from Alomone Labs, Ltd. (Jerusalem, Israel). Monoclonal rabbit anti-TrKB (1:1,000; cat. no. 4603S), monoclonal rat anti-phosphorylated (p)-TrKB (tyrosine $\left.^{816}\right)(1: 1,000$; cat. no. 4168S), monoclonal rabbit anti-p75 ${ }^{\mathrm{NTR}}(1: 1,000$; cat. no. 4201S) and monoclonal rabbit anti- $\beta$-actin $(1: 1,000$; cat. no. $4970 \mathrm{~S})$ primary antibodies were obtained from Cell Signaling Technology, Inc. (Danvers, MA, USA). The horseradish peroxidase (HRP)-conjugated monoclonal goat secondary antibodies [anti-rabbit IgG $(1: 5,000$; cat. no. 7074P2) and anti-rat IgG (1:5,000; cat. no. 7077S)] were also obtained from Cell Signaling Technology, Inc. All other chemicals used, unless otherwise stated, were obtained from Beyotime Institute of Biotechnology (Haimen, China).

Animals. Thirty APP/PS1 double-transgenic mice [B6C3-Tg(APPswe,PSEN1dE9)85Dbo/MmJNju], aged 3 months, and their 10 wild-type (WT) littermates (age, 3 months) were purchased from Nanjing Biomedical Research Institute of Nanjing University (Nanjing, China). The mice were housed five per cage in a controlled environment (22-25 $\mathrm{C} ; 55 \%$ relative humidity; 12 -h light/dark cycle) with access to food and water ad libitum. They were maintained in a specific pathogen-free environment for two months prior to being sacrificed. The experiments were approved by the Institutional Animal Care and Use Committee of Fujian University of Traditional Chinese Medicine, and were strictly in accordance with international ethical guidelines and the National Institutes of Health Guide for the Care and Use of Laboratory Animals (16).

Animal grouping and treatment. Thirty APP/PS1 double-transgenic mice (that exhibit early onset of $\mathrm{A} \beta$ deposition, followed by neuronal loss and cognitive impairment) were randomly and evenly divided into three groups $(n=10)$ as follows: i) the APP/PS1 double-transgenic mice control group (APP/PS1); ii) the EA at the Baihui (DU20) acupoint group (APP/PS1 + DU20); and iii) the EA at non-acupoint group (APP/PS1 + NA). Another $10 \mathrm{WT}$ littermates (that do not exhibit early onset of $\mathrm{A} \beta$ deposition, neuronal loss and cognitive impairment) were designated as the aging control group (WT group). In the APP/PS1 + DU20 group, mice were administered EA for 30 min daily for 4 weeks. The acupuncture needles (diameter, $0.3 \mathrm{~mm}$ ) were inserted at a depth of 2-3 mm into the Baihui (DU20) acupoint, which is located at the intersection of the sagittal midline and the line linking the two ears. Stimulation was generated using EA apparatus (model G6805; Suzhou Medical Appliance Factory, Shanghai, China) and the stimulation parameters were set as disperse waves of 1 and $20 \mathrm{~Hz}$. In the APP/PS1 + NA group, a non-acupoint (the area below the costal region, $2 \mathrm{~cm}$ superior to the posterior superior iliac spine and $\sim 3 \mathrm{~cm}$ lateral to the spine) was punctured and stimulated for $30 \mathrm{~min}$ daily for 4 weeks. The two groups (APP/PS1 + DU20 and APP/PS1 + NA) received treatment with the same needles, stimulation parameters and EA apparatus. The APP/PS1 and WT groups did not receive any EA treatment.

Morris water maze test. All the mice were subjected to the Morris water maze test, including orientation navigation and space exploration trials following 4 weeks of treatment. This was conducted to investigate spatial learning and memory ability, as previously described (17). The water maze apparatus (Chinese Academy of Sciences, Beijing, China) is a stainless steel circular water tank (diameter, $120 \mathrm{~cm}$; height, $50 \mathrm{~cm}$ ) equipped with a platform (diameter, $10 \mathrm{~cm}$ ) placed in the third quadrant and submerged $2 \mathrm{~cm}$ below the surface of the water. A video camera attached to a computer was placed above the center of the tank to record and analyze the trajectory of the mice. During the orientation navigation trials, each mouse was placed in the water at each of four equidistant locations to the platform. When a mouse arrived at the platform within the $90 \mathrm{sec}$ time restriction and remained on it for $5 \mathrm{sec}$, it was considered to have found the platform. If the mouse failed to find the platform within $90 \mathrm{sec}$, it would be removed from the water and placed on the platform for $10 \mathrm{sec}$. The computer recorded the length of the route and the time it took the mouse to find the platform. The orientation navigation trials were repeated for four days. On the fifth day, the space exploration test was performed to assess memory consolidation. In this trial, the platform was removed from the tank, and the mice were allowed to swim freely for $90 \mathrm{sec}$. The novel start position was located $180^{\circ}$ from the original platform position to ensure 

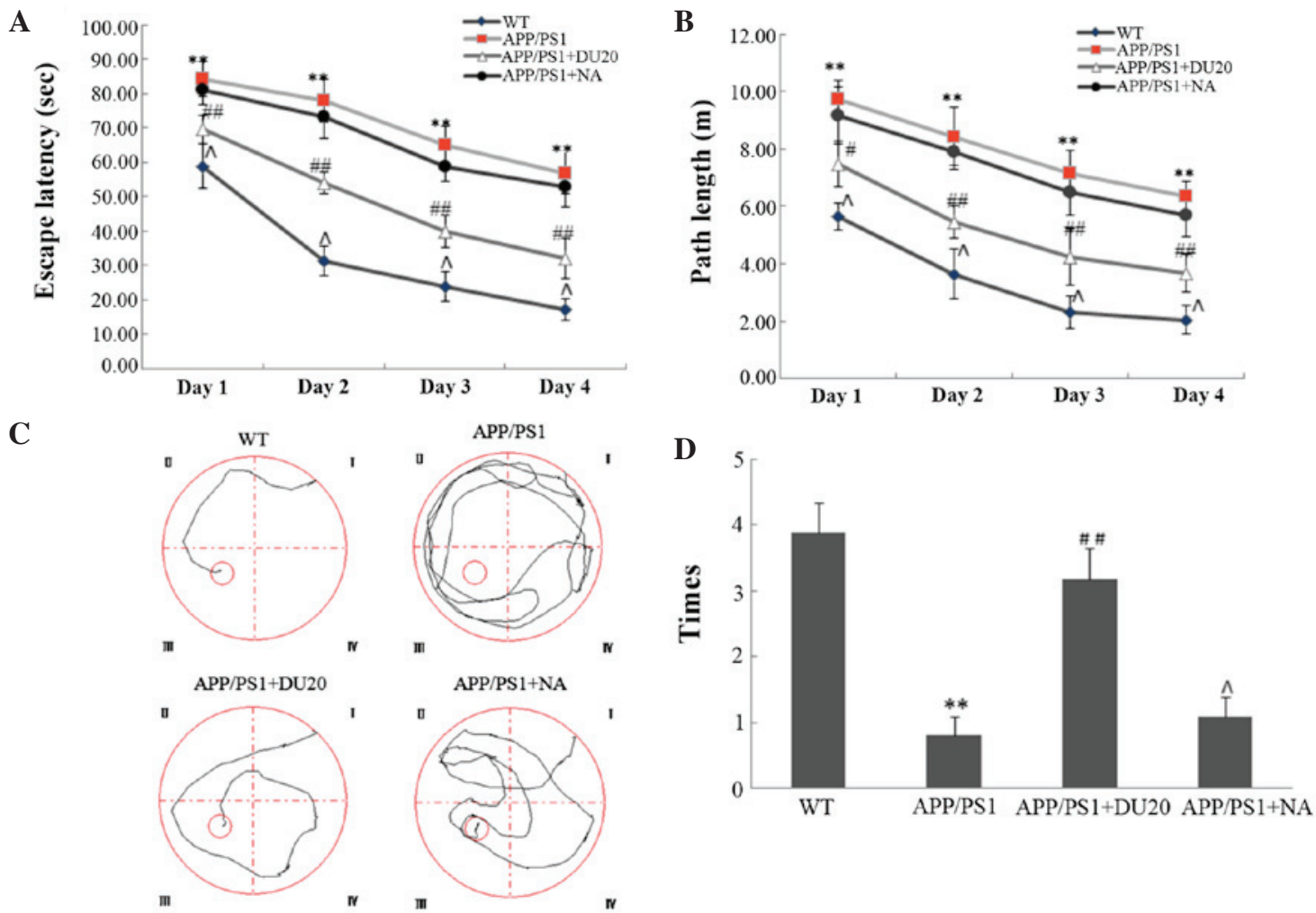

Figure 1. Effect of electroacupuncture at the Baihui (DU20) acupoint on cognitive impairment in APP/PS1 mice. (A and B) The escape latency time and path length during the orientation navigation test following 4 weeks of treatment. (C) Representative tracing images of the space exploration test. The red circle in quadrant III represents the platform location. (D) The number of times the rats passed through the original position of the platform. ${ }^{* *} \mathrm{P}<0.01 \mathrm{vs.} \mathrm{the} \mathrm{WT} \mathrm{group;}$ ${ }^{\#} \mathrm{P}<0.05,{ }^{\# \#} \mathrm{P}<0.01$ and ${ }^{\wedge} \mathrm{P}>0.05$ vs. the APP/PS1 group. Each group, $\mathrm{n}=10$. WT, wild-type; NA, non-acupoint.

A WT

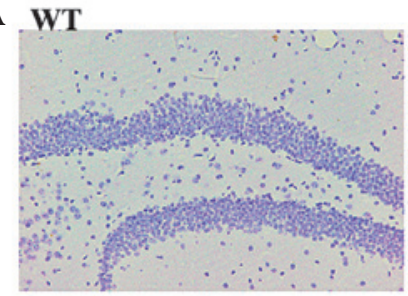

APP/PS1+DU20

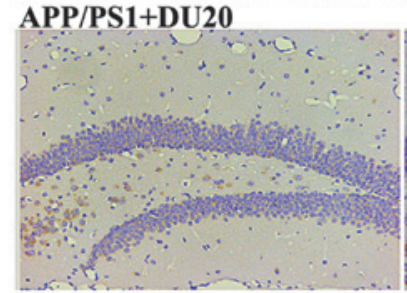

$\mathbf{B}$

จิ์

120

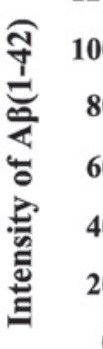

APP/PS1

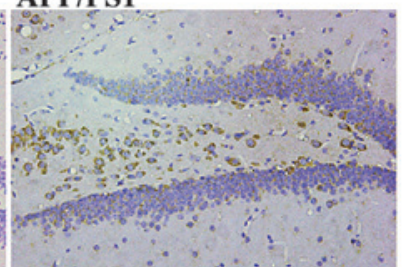

APP/PS1+NA
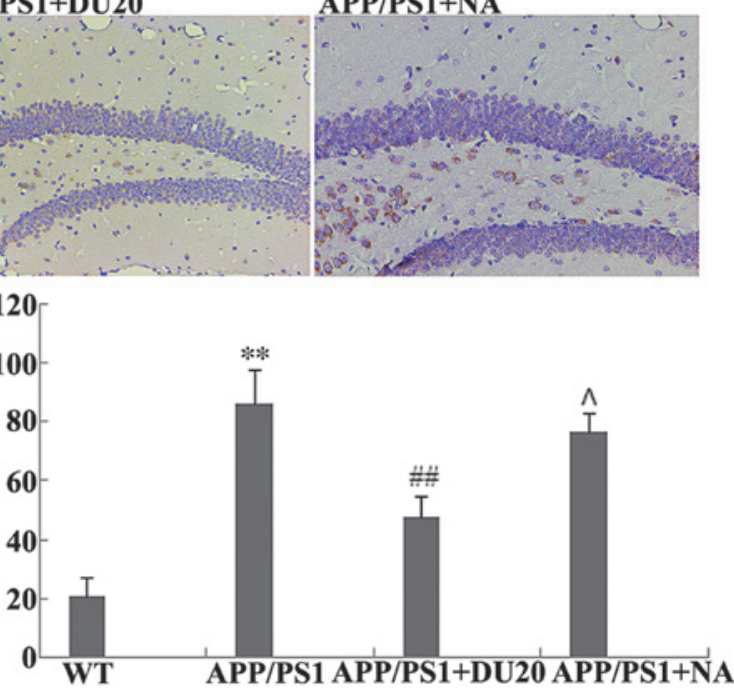

Figure 2. Effect of electroacupuncture at the Baihui (DU20) acupoint on $A \beta(1-42)$ deposition in the hippocampus of APP/PS1 mice. (A) Immunohistochemical staining for $A \beta(1-42)$ in the hippocampus of each group (magnification, $x 400$ ). (B) The intensity of staining for $A \beta(1-42)$ in each group. ${ }^{* *} \mathrm{P}<0.01$ vs. the WT group, ${ }^{\# /} \mathrm{P}<0.01$ and ${ }^{\wedge} \mathrm{P}>0.05$ vs. the APP/PS1 group. Each group, $\mathrm{n}=5$. A $\beta, \beta$ amyloid; WT, wild-type; NA, non-acupoint. that the spatial preference was a reflection of the memory of the goal location, rather than for a specific swim path. The frequency that each mouse crossed the center of the quadrant (where the platform was previously located) was recorded.

TUNEL staining. The mice were anesthetized via injection of $10 \%$ chloral hydrate $(0.03 \mathrm{ml} / 100 \mathrm{~g}$ body weight; Shanghai Reagent Factory, Shanghai, China) and transcardially perfused with a $0.9 \%$ saline $(\mathrm{NaCl})$ solution and $4 \%$ paraformaldehyde via the left ventricle, and subsequently the brain was removed. The tissue samples were fixed in cold $4 \%$ paraformaldehyde at $4^{\circ} \mathrm{C}$ overnight, embedded in paraffin (Tianjin Fuchen Chemical Reagent Factory, Tianjin, China) and cut into 5- $\mu \mathrm{m}$ sections. In situ apoptosis was analyzed using a TUNEL assay kit according to the manufacturer's protocols. The nuclei of all the cells were visualized by DAPI staining and apoptotic cells were detected using a confocal fluorescence microscope (LSM710; Carl Zeiss AG, Oberkochen, Germany). Apoptotic cells were counted at four randomly selected microscopic fields (magnification, $\mathrm{x} 400$ ) and the apoptotic rate was measured as a ratio of the TUNEL-positive cells to the total number of cells.

Immunohistochemistry (IHC). IHC was performed on the $5-\mu \mathrm{m}$ paraffin sections. A $\beta(1-42)$ levels were examined with DAB kits according to the manufacturer's protocols. The sections were incubated in 3\% hydrogen peroxide and normal serum at $37^{\circ} \mathrm{C}$ for $10 \mathrm{~min}$ to block the non-specific protein binding. Sections were incubated with primary anti-A $\beta(1-42)$ antibody (1:200) at $4^{\circ} \mathrm{C}$ overnight and subsequently incubated 


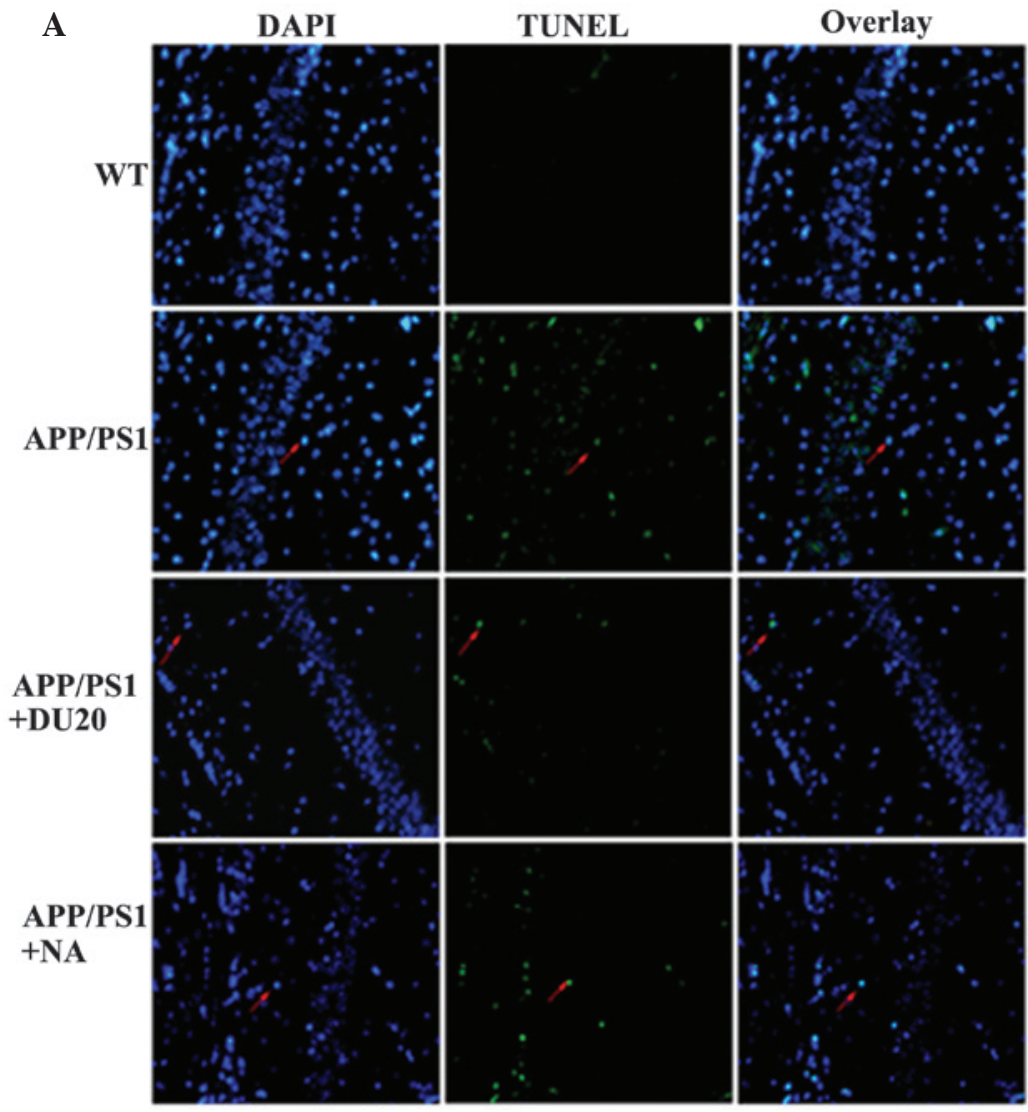

B

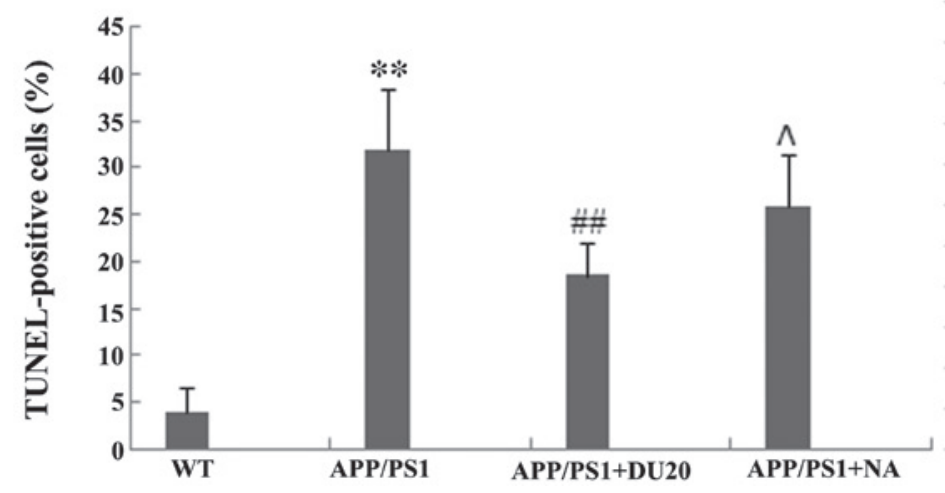

Figure 3. Effect of electroacupuncture at the Baihui (DU20) acupoint on cell apoptosis using a TUNEL assay. (A) TUNEL staining in the hippocampus of each group (magnification, $\mathrm{x} 400$ ). Red arrows indicate TUNEL-positive cells. (B) The percentage of TUNEL-positive cells from each group. ${ }^{* *} \mathrm{P}<0.01 \mathrm{vs}$. the WT group; ${ }^{\# \#} \mathrm{P}<0.01$ and ${ }^{\wedge} \mathrm{P} 0.05$ vs. the APP/PS1 group. Each group, $\mathrm{n}=5$. TUNEL, terminal deoxynucleotidyl transferase dUTP nick end labeling; WT, wild-type; NA, non-acupoint.

with secondary antibody. A $\beta(1-42)$-positive cells were stained brown and hematoxylin was used to visualize the nuclei of all cells. Images of $A \beta(1-42)$ deposition in the hippocampus were captured using an optic microscope (DFC310 FX; Leica Microsystems, Inc., Buffalo Grove, IL, USA) and analyzed with an image analysis system (Image-Pro Plus, version 6.0; Motic China Group Co., Ltd., Xiamen, China). The density of $A \beta(1-42)$ deposition (the percentage of positively-stained brown cells) was determined by subtracting the background density and non-specific binding. The software was used to perform the semi-quantitative evaluation.

Western blot analysis. Protein was extracted from the hippocampus and separated by electrophoresis (90 V for $30 \mathrm{mins}$ ) on $12 \%$ SDS-PAGE gels (Bio-Rad Laboratories, Inc., Hercules,
CA, USA). Proteins were transferred onto polyvinylidene fluoride membranes (EMD Millipore Billerica, MA, USA). The membranes were blocked for $2 \mathrm{~h}$ with $5 \%$ nonfat dried milk at room temperature and incubated with antibodies against BDNF $(1: 1,000)$, proBDNF $(1: 400)$, TrkB $(1: 1,000)$, p-TrkB $(1: 1,000)$, p $75^{\mathrm{NTR}}(1: 1,000)$, and $\beta$-actin $(1: 5,000)$ at $4^{\circ} \mathrm{C}$ overnight and subsequently incubated with HRP-conjugated secondary antibody $(1: 5,000)$ for $1 \mathrm{~h}$. The protein bands were visualized with enhanced chemiluminescence and imaged with the Bio-Image Analysis system (Bio-Rad Laboratories, Inc.). The ratios of protein band intensities to $\beta$-actin were determined and subsequently normalized to the WT group.

Statistical analysis. All data were analyzed using the SPSS package, version 18.0 (SPSS, Inc., Chicago, IL, USA). 
A

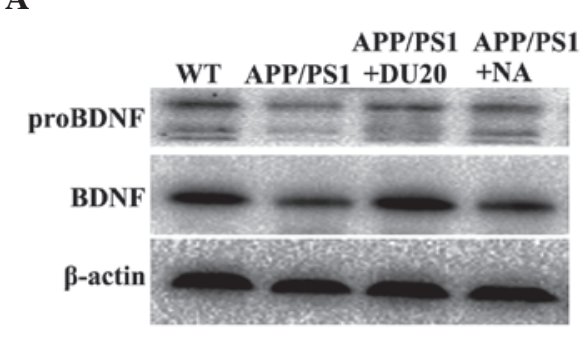

C

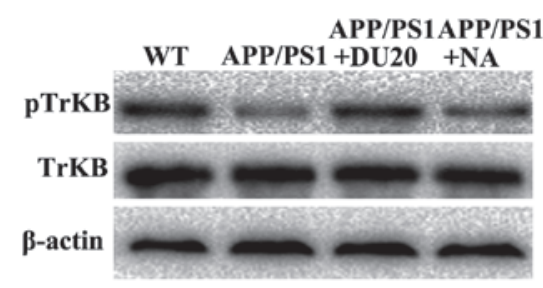

$\mathbf{E}$

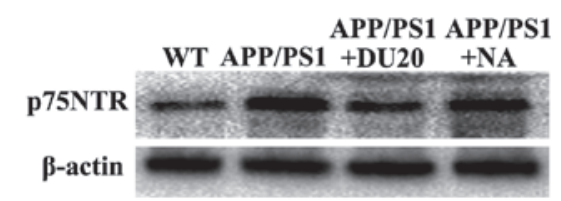

B

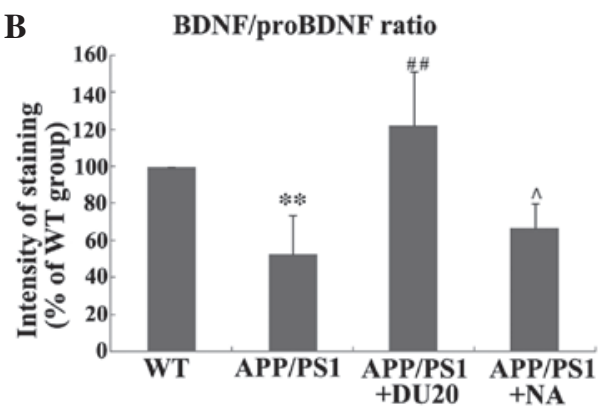

D
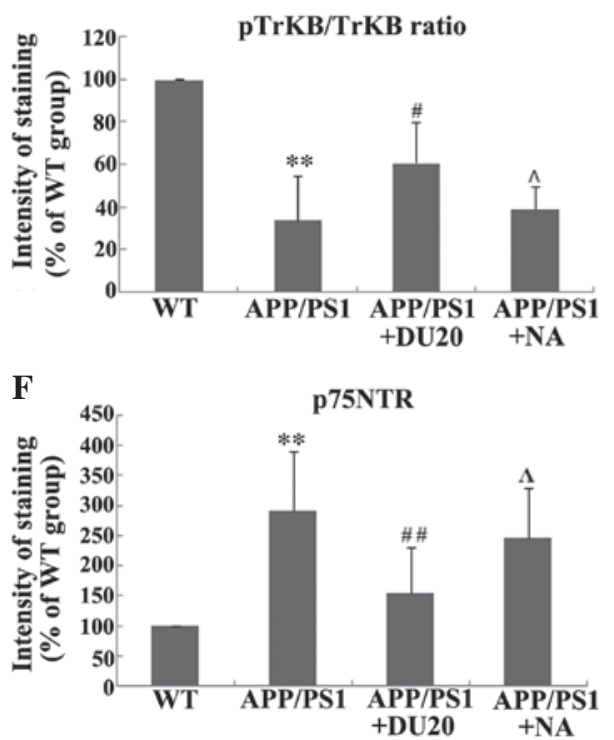

Figure 4. Effect of electroacupuncture at the Baihui (DU20) acupoint on the expression and processing of BDNF. (A) Representative western blot demonstrating protein expression levels of BDNF and proBDNF in the hippocampus from each group. (B) Ratio of BDNF to proBDNF normalized to the WT group. (C) Representative western blot indicating the levels of TrkB and p-TrkB. (D) Ratio of p-TrkB vs. total TrkB normalized to the WT group. (E) Representative western blot demonstrating the levels of p75 ${ }^{\mathrm{NTR}}$ in each group. (F) Expression levels of p75 ${ }^{\mathrm{NTR}}$ normalized to the WT group. ${ }^{* *} \mathrm{P}<0.01$ vs. the WT group; ${ }^{\#} \mathrm{P}<0.05,{ }^{\# \#} \mathrm{P}<0.01$ and ${ }^{\wedge} \mathrm{P}>0.05$ vs. the APP/PS1 group. Each group, $\mathrm{n}=5$. BDNF, brain-derived neurotrophic factor; WT, wild-type; NA, non-acupoint; TrkB, tropomyosin receptor kinase $\mathrm{B} ; \mathrm{p} 75^{\mathrm{NTR}}$, $\mathrm{p} 75$ neurotrophin receptor; $\mathrm{p}$, phosphorylated.

Quantitative data are presented as the mean \pm standard error. Student's t-tests and one-way analysis of variance were used to assess statistical differences among groups and $\mathrm{P}<0.05$ was considered to indicate a statistically significant difference.

\section{Results}

EA at the Baihui (DU20) acupoint ameliorates cognitive impairment in APP/PS1 mice. To evaluate the effect of EA at the Baihui (DU20) acupoint on cognitive impairment in APP/PS1 mice, the Morris water maze test was performed. In the orientation navigation test, the APP/PS1 group demonstrated increased escape latency (the time it took the mouse to find the platform) and longer path length (Fig. 1A and B) compared with the WT group $(\mathrm{P}<0.01)$. EA at the Baihui (DU20) acupoint treatment significantly reduced the escape latency and the path length compared with the APP/PS1 group $(\mathrm{P}<0.01$; Fig. 1A and B). Furthermore, in the space exploration test (where the platform was removed) the APP/PS1 mice passed through the original position of the platform fewer times than the WT group $(\mathrm{P}<0.01$; Fig. $1 \mathrm{C}$ and $\mathrm{D})$. In the APP/PS1 + DU20 group, the frequency that the mice crossed the position of the platform was significantly increased compared with the APP/PS1 group ( $\mathrm{P}<0.01$; Fig. $1 \mathrm{C}$ and $\mathrm{D})$. However, EA at a non-acupoint (APP/PS1 + NA) resulted in similar findings for the APP/PS1 mice (Fig. 1). Collectively, these data suggest that EA at the Baihui (DU20) acupoint ameliorates cognitive impairment in APP/PS1 mice.

EA at the Baihui (DU20) acupoint decreases overexpression of $A \beta(1-42)$ in the hippocampus of APP/PS1 mice. A typical pathological feature of $\mathrm{AD}$ is the overexpression of $\mathrm{A} \beta(1-42)$. Therefore, the effect of EA at the Baihui (DU20) acupoint on $\mathrm{A} \beta(1-42)$ deposition in the hippocampus of APP/PS1 mice was investigated. As demonstrated in Fig. 2, the APP/PS1 group exhibited a significant increase in $\mathrm{A} \beta(1-42)$ deposition $(\mathrm{P}<0.01$ versus the WT group), which was attenuated by $\mathrm{EA}$ at the Baihui (DU20) acupoint (APP/PS1 + DU20), although not with stimulation of a non-acupoint (APP/PS1 + NA). These results demonstrate that EA at the Baihui (DU20) acupoint effectively decreases the deposition of $A \beta(1-42)$ in the hippocampus.

EA at the Baihui (DU20) acupoint inhibits neuronal apoptosis in the hippocampus of APP/PS1 mice. Neuronal loss in the hippocampus is another important pathological feature of $\mathrm{AD}$. In order to investigate the effects of EA at the Baihui 
(DU20) acupoint on neuronal apoptosis, a TUNEL assay was conducted. The percentage of TUNEL-positive cells was significantly increased in the hippocampus of the APP/PS1 group ( $\mathrm{P}<0.01$ versus the WT group; Fig. 3).

EA at the Baihui (DU20) acupoint, but not at a non-acupoint, reverses the aberrant cell death observed in the APP/PS1 mice. EA at the Baihui (DU20) acupoint altered the expression and processing of BDNF in the hippocampus of APP/PS1 mice. As BDNF is important in the pathogenesis of $\mathrm{AD}$, the effect of EA at the Baihui (DU20) acupoint on the expression and processing of BDNF in the hippocampus of APP/PS1 mice was investigated. EA at the Baihui (DU20) acupoint was observed to significantly increase the expression levels of BDNF and proBDNF ( $\mathrm{P}<0.01$ vs. the WT group; Fig. 4A). Furthermore, EA at the Baihui (DU20) acupoint significantly increased the BDNF/proBDNF ratio in the APP/PS1 + DU20 group ( $\mathrm{P}<0.01$ vs. the WT group; Fig. $4 \mathrm{~B})$. In addition, $\mathrm{p}-\mathrm{TrkB}$ was upregulated (Fig. 4C and D), while the expression level of $\mathrm{p} 75^{\mathrm{NTR}}$ was decreased in the APP/PS1 + DU20 group $(\mathrm{P}<0.01$ vs. the WT group; Fig. 4D and E). These data suggest that EA at the Baihui (DU20) acupoint may exert neuroprotective effects via modulation of the expression and processing of BDNF.

\section{Discussion}

$\mathrm{AD}$ is a prevalent neurodegenerative diseases characterized by progressive deterioration of cognition and memory (18). At present, the majority of therapeutic agents used to clinically treat AD target the cholinergic system, histaminergic system or 5-hydroxy tryptamine receptor $(19,20)$. These therapeutic agents improve the symptoms associated with AD and delay progression to a certain extent, however they do not stop or reverse the disease progression (21). Acupuncture at the Baihui (DU20) acupoint has long been used in China to treat cognitive impairment and may be a promising treatment strategy for AD. However, until now, the efficacy of acupuncture at the Baihui (DU20) acupoint has only been evaluated in animal studies and its underlying mechanism of action requires elucidation. In the present study, EA at the Baihui (DU20) acupoint was observed to reverse the cognitive impairments in APP/PS1 transgenic mice via modulation of BDNF expression and processing.

The APP/PS1 transgenic mouse model mimics the pathology of $\mathrm{AD}$ by combining two strategies to elevate $\mathrm{A} \beta$ levels, overexpression of the gene encoding the human amyloid precursor protein and the mutant presenilin-1 gene, which impairs amyloid protein processing, resulting in elevated A $\beta(1-42)$ levels (22). APP/PS1 transgenic mice exhibit early onset and a high degree of $A \beta$ deposition, followed by neuronal loss and cognitive impairment (23). Thus, are considered to be reliable and effective models that are widely used in AD research (24). In the current study, APP/PS1 mice demonstrated increases in parameters assessed by the Morris water maze test, including higher escape latency, longer path length, and fewer times passing through the platform, as well as a notable increase in $A \beta(1-42)$ deposition and significant neuronal loss in the hippocampus when compared with WT mice. Notably, EA at the Baihui (DU20) acupoint (APP/PS1 + DU20) significantly reversed all of these aberrations. However, EA at a non-acupoint (APP/PS1 + NA) revealed similar results to the APP/PS1 mice. Therefore, EA at the Baihui (DU20) acupoint is considered to be an effective method for treatment of the cognitive impairments observed in APP/PS1 transgenic mice.

To further investigate the neuroprotective effects of EA at the Baihui (DU20) acupoint in APP/PS1 mice, the expression and processing of BDNF was investigated. BDNF is a major regulator of synaptic plasticity, neuronal survival and differentiation, as well as higher order processes, including learning, memory and behavior (25). It is well established that BDNF is important in the pathogenesis of AD $(26,27)$. Numerous studies have demonstrated that BDNF administration exerts substantial protective effects on crucial neuronal circuits involved in AD by preventing cell death and neuronal atrophy, thereby ameliorating cognitive and behavioral deficits $(28,29)$. In the present study, EA at the Baihui (DU20) acupoint was observed to significantly enhance the expression of BDNF and proBDNF in APP/PS1 mice. These results demonstrate that EA at the Baihui (DU20) acupoint may exert neuroprotective effects by increasing expression levels of BDNF. However, the mechanism by which EA increases the expression of BDNF so as to exert neuroprotective effects remains to be elucidated. BDNF is synthesized as a precursor protein, proBDNF that is post-translationally cleaved to produce mBDNF. Notably, proBDNF exerts opposing biological effects to BDNF signaling (30). ProBDNF binds to $\mathrm{p} 75^{\mathrm{NTR}}$ resulting in the activation of apoptosis-associated signaling pathways and facilitating long-term depression in the hippocampus $(31,32)$. Therefore, processing of the BDNF protein from proBDNF to mBDNF appears to be important in the regulation of cellular functions. The outcomes of the processing can be reflected by the relative expression level of BDNF and proBDNF (the BDNF/proBDNF ratio), which is key in regulating cellular functions. In the present study, EA at the Baihui (DU20) acupoint increased the $\mathrm{BDNF} /$ proBDNF ratio. Furthermore, the receptors of BDNF and proBDNF were investigated and it was observed that EA at the Baihui (DU20) acupoint upregulated the expression of $\mathrm{p}$-TrkB and decreased the expression level of $\mathrm{p} 75^{\mathrm{NTR}}$ in the APP/PS1 mice. The results indicate that EA at the Baihui (DU20) acupoint influenced the modulation and processing of the BDNF protein from proBDNF to mBDNF, and eventually enhanced the expression levels of mBDNF. Collectively, these data suggest that EA at the Baihui (DU20) acupoint may exert neuroprotective effects by adjusting the expression and processing of BDNF.

In conclusion, results from the present study demonstrate the efficacy of EA at the Baihui (DU20) acupoint in APP/PS1 transgenic mice, and indicate that EA at the Baihui (DU20) acupoint may exert neuroprotective effects by modulating the expression and processing of BDNF. These results suggest that EA at the Baihui (DU20) acupoint may serve as a promising treatment strategy for AD.

\section{Acknowledgements}

The authors would like to thank Clarity Manuscript Consultants, LLC for their editorial assistance with this manuscript. 


\section{References}

1. Mayeux R: Clinical practice. Early Alzheimer's disease. N Engl J Med 362: 2194-2201, 2010.

2. Querfurth HW and LaFerla FM: Alzheimer's disease. N Engl J Med 362: 329-344, 2010.

3. Serrano-Pozo A, Frosch MP, Masliah E and Hyman BT: Neuropathological alterations in Alzheimer disease. Cold Spring Harb Perspect Med 1: a006189, 2011.

4. Phillips HS, Hains JM, Armanini M, Laramee GR, Johnson SA and Winslow JW: BDNF mRNA is decreased in the hippocampus of individuals with Alzheimer's disease. Neuron 7: 695-702, 1991.

5. Lee J, Fukumoto H and Orne J, Klucken J, Raju S, Vanderburg CR, Irizarry MC, Hyman BT and Ingelsson M: Decreased levels of BDNF protein in Alzheimer temporal cortex are independent of BDNF polymorphisms. Exp Neurol 194: 91-96, 2005.

6. Peng S, Wuu J, Mufson EJ and Fahnestock M: Precursor form of brain-derived neurotrophic factor and mature brain-derived neurotrophic factor are decreased in the pre-clinical stages of Alzheimer's disease. J Neurochem 93: 1412-1421, 2005.

7. Weinstein G, Beiser AS and Choi SH, Preis SR, Chen TC, Vorgas D, Au R, Pikula A, Wolf PA, DeStefano AL, et al: Serum brain-derived neurotrophic factor and the risk for dementia: The Framingham Heart Study. JAMA Neurol 71: 55-61, 2014.

8. Barker PA: Whither proBDNF? Nat Neurosci 12: 105-106, 2009.

9. Greenberg ME, Xu B, Lu B and Hempstead BL: New insights in the biology of BDNF synthesis and release: Implications in CNS function. J Neurosci 29: 12764-12767, 2009.

10. Yang J, Harte-Hargrove LC, Siao CJ, Marinic T, Clarke R, Ma Q, Jing D, Lafrancois JJ, Bath KG, Mark W, et al: proBDNF negatively regulates neuronal remodeling, synaptic transmission, and synaptic plasticity in hippocampus. Cell Reports 7: 796-806, 2014

11. Minichiello L: TrkB signalling pathways in LTP and learning. Nat Rev Neurosci 10: 850-860, 2009.

12. Arancibia S, Silhol M, Moulière F, Meffre J, Höllinger I, Maurice T and Tapia-Arancibia L: Protective effect of BDNF against beta-amyloid induced neurotoxicity in vitro and in vivo in rats. Neurobiol Dis 31: 316-326, 2008.

13. Kemppainen S, Rantamäki $\mathrm{T}$ and Jerónimo-Santos A, Lavasseur G, Autio H, Karpova N, Kärkkäinen E, Stavén S, Vicente Miranda H, Outeiro TF, et al: Impaired TrkB receptor signaling contributes to memory impairment in APP/PS1 mice. Neurobiol Aging 33, 2012 (Epub ahead of print).

14. Xu ZQ, Li J, Deng J, Jiang XJ and Zhou HD: Effects of proBDNF on cell proliferation and differentiation in hippocampal dentate gyrus in Alzheimer's disease rat model. Chin Med J 90: 1353-1356, 2010 (In Chinese).

15. Feng $X$, Yang $S$ and Liu J, Huang J, Peng J, Lin J, Tao J and Chen L: Electroacupuncture ameliorates cognitive impairment through inhibition of NF- $\kappa \mathrm{B}$-mediated neuronal cell apoptosis in cerebral ischemia-reperfusion injured rats. Mol Med Rep 7: 1516-1522, 2013
16. International Ethical Guidelines for Biomedical Research Involving Human Subjects. WHO/CIOMS, Geneva, 1993.

17. Vorhees CV and Williams MT: Morris water maze: Procedures for assessing spatial and related forms of learning and memory. Nat Protoc 1: 848-858, 2006.

18. Selkoe DJ: Alzheimer's disease is a synaptic failure. Science 298: 789-791, 2002.

19. Haas HL, Sergeeva OA and Selbach O: Histamine in the nervous system. Physiol Rev 88: 1183-1241, 2008.

20. Brioni JD, Esbenshade TA, Garrison TR, Bitner SR and Cowart MD: Discovery of histamine H3 antagonists for the treatment of cognitive disorders and Alzheimer's disease. J Pharmacol Exp Ther 336: 38-46, 2011.

21. Ballard C, Gauthier S, Corbett A, Brayne C, Aarsland D and Jones E: Alzheimer's disease. Lancet 377: 1019-1031, 2011.

22. Radde R, Bolmont T, Kaeser SA, Coomaraswamy J, Lindau D, Stoltze L, Calhoun ME, Jäggi F, Wolburg H, Gengler S, et al: Abeta42-driven cerebral amyloidosis in transgenic mice reveals early and robust pathology. EMBO Rep 7: 940-946, 2006.

23. Borchelt DR, Ratovitski T, van Lare J, Lee MK, Gonzales V, Jenkins NA, Copeland NG, Price DL and Sisodia SS: Accelerated amyloid deposition in the brains of transgenic mice coexpressing mutant presenilin 1 and amyloid precursor proteins. Neuron 19: 939-945, 1997

24. Bilkei-Gorzo A: Genetic mouse models of brain ageing and Alzheimer's disease. Pharmacol Ther 142: 244-257, 2014.

25. Binder DK and Scharfman HE: Brain-derived neurotrophic factor. Growth Factors 22: 123-131, 2004.

26. Cosker KE, Courchesne SL and Segal RA: Action in the axon: generation and transport of signaling endosomes. Curr Opin Neurobiol 18: 270-275, 2008.

27. Schindowski K, Belarbi K and Buée L: Neurotrophic factors in Alzheimer's disease: role of axonal transport. Genes, Brain Behav 7 (suppl 1): 43-56, 2008

28. Tapia-Arancibia L, Aliaga E, Silhol M and Arancibia S: New insights into brain BDNF function in normal aging and Alzheimer disease. Brain Res Rev 59: 201-220, 2008.

29. Castello NA, Nguyen MH, Tran JD, Cheng D, Green KN and LaFerla FM: 7,8-Dihydroxyflavone, a small molecule TrkB agonist, improves spatial memory and increases thin spine density in a mouse model of Alzheimer disease-like neuronal loss. PLoS One 9: e91453, 2014.

30. Lu B, Pang PT and Woo NH: The yin and yang of neurotrophin action. Nat Rev Neurosci 6: 603-614, 2005.

31. Woo NH, Teng HK, Siao CJ, Chiaruttini C, Pang PT, Milner TA, Hempstead BL and Lu B: Activation of p75NTR by proBDNF facilitates hippocampal long-term depression. Nat Neurosci 8: 1069-1077, 2005.

32. Teng HK, Teng KK, Lee R, Wright S, Tevar S, Almeida RD, Kermani P, Torkin R, Chen ZY, Lee FS, et al: ProBDNF induces neuronal apoptosis via activation of a receptor complex of p75NTR and sortilin. J Neurosci 25: 5455-5463, 2005. 